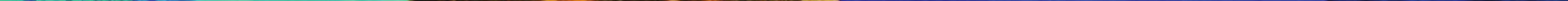




\section{Situación de los jovenes en edad de trabajar en el municipio de Colomoncagua Intibucá}

María Juana JaVier*

Mario Trejo**

Seylin Margarita Hernández ***

RESUMEN. Honduras es un país que dentro de su estructura población cuenta con un alto porcentaje de población joven. Esta misma tendencia se refleja a nivel de los municipios.

Uno de los problemas de este grupo poblacional está determinado por las escasas oportunidades laborales y educativas sobre todo entre la población rural y de aquellos municipios postergados del país.

La investigación refleja la situación socioeconómica de la población joven de 11 a 29 años, del municipio de Colomoncagua, Intibucá, identificando las actividades económicas a las que se dedican los jóvenes en el municipio, los y las jóvenes que se encuentran en edades de trabajar y no estudian ni trabajan y las organizaciones estatales y privadas que han apoyado a la población joven del municipio de Colomoncagua.

Los resultados ponen en alerta a las autoridades locales y nacionales sobre la necesidad de implementar proyectos y programas sociales de beneficio para los y las jóvenes de tal manera que permitan crear oportunidades educativas y laborales que favorezca su nivel de vida.

Palabras clave: jóvenes, mercado laboral, PEA.

\section{PRESENTACIÓN}

El presente documento es el resultado de la investigación realizada en el municipio de Colomoncagua, departamento de Intibucá, respecto a la situación de los y la jóvenes comprendidos entre 11 y 29 años de edad.
La investigación se desarrolló a partir de los conocimientos adquiridos en el Diplomado Objetivos de Desarrollo del Milenio, realizado en la ciudad de La Esperanza y en el contexto de la organización de células de investigación que funcionan en el Departamento de Valle e Intibucá. La constitución de células en estas zonas del país tiene como propósito investigar temas que se constituyan en un aporte a la comunidad local y nacional.

El documento pretende constituirse como una herramienta útil de consulta para la población, gobierno municipal, las ONGs y en particular para el personal responsable de la toma de decisiones en la formulación de proyectos dirigidos a jóvenes.

El contenido de este trabajo da cuenta de las características sociodemográficas, relacionadas con la población joven de Colomoncagua. La información utilizada proviene de fuente primaria obtenida mediante la aplicación de una encuesta en diferentes comunidades del municipio, tomando en cuenta a todos los jóvenes entre 11 a 29 años de edad.

\section{JUSTIFICACIÓN}

Según el Censo de Población y Vivienda del 2001 realizado por el Instituto Nacional de Estadística el mayor porcentaje de la población está constituido por población joven. Casi dos tercios de la población total registrada en ese año tenían menos de 25 años por lo que este grupo

Recibido: noviembre 2009/ Aceptado y versión final: febrero 2010.

* Diplomada en Objetivos de Desarrollo del Milenio con Énfasis en Análisis Socio demográfico, jjavier@yahoo.es

** Diplomado en Objetivos de Desarrollo del Milenio con Énfasis en Análisis Sociodemográfico, m.trejo@yahoo.es

*** Lic. en Economía Agrícola CURLA- UNAH Maestrante de Demografía Social hseylin@yahoo.com 
tiene un peso poblacional que debe considerarse dentro de las políticas de Estado y los proyectos de desarrollo.

En el caso de Colomoncagua el 60\% de la población censada en el 2001 era menor de 20 años, lo que permite afirmar que casi dos tercios de la población del municipio está constituido por gente muy joven. Sin embargo, esta población presenta condiciones de precariedad que impiden el desarrollo pleno de sus potencialidades.

Una de las limitantes que los jóvenes del municipio presentan está relacionada con el acceso a fuentes de trabajo y a recursos económicos que les permitan un nivel de vida digno, esta situación en gran parte de los casos les obliga a migrar en busca de mejores oportunidades laborales.

Las razones anteriores motivan el desarrollo de la presente investigación cuyo objetivo se orienta describir la situación socioeconómica de la población joven de 11 a 29 años en el municipio de Colomoncagua, Intibucá. Este estudio permitirá tener una visión de la problemática social y laboral que presenta este grupo poblacional.

Al conocer la situación actual en la que se encuentran los jóvenes se identificaron características propias de la juventud, ¿qué están haciendo actualmente?, además se verificó la falta de oportunidades, y esto representa un riesgo para la sociedad porque los jóvenes son el eje de tres de los principales problemas que se presentan a nivel nacional: el desempleo, la delincuencia y la fragilidad democrática.

\section{OBJETIVOS DE LA INVESTIGACIÓN}

\subsection{Objetivo General}

- Describir la situación socioeconómica de la población joven de 11 a 29 años, del municipio de Colomoncagua, Intibucá.

\subsection{Objetivos Específicos}

- Identificar las actividades económicas a las que se dedican los jóvenes en el municipio de Colomoncagua.

- Identificar la cantidad de jóvenes que se encuentran en edades de trabajar y no estudian ni trabajan.

- Identificar qué organizaciones estatales y privadas han apoyado a la población joven del municipio de Colomoncagua.

\section{METODOLOGÍA}

Metodológicamente la investigación propuesta se realizó en varias etapas:

a. Revisión bibliográfica que permitió indagar el tema de juventud

b. Selección del tema de investigación: se escogió el tema basado en el peso poblacional que tiene la juventud en el municipio y la carencia de estudios que aborden la problemática de este grupo poblacional.

c. Elaboración de instrumento de recolección de datos que consistió en una Encuesta elaborada en base a los objetivos de investigación propuestos y aplicada a jóvenes comprendidos entre los 11 a 29 años de edad.

d. Marco muestral: se trabajó con las 10 aldeas del municipio, 30 encuestas por aldea.

e. Aplicación del instrumento, este se aplicó en todas las aldeas del municipio en un período de tres semanas.

f. Elaboración de base de datos; los datos fueron procesados en SPSS versión 15 al igual que las tablas de salida. Los gráficos se elaboraron en excell.

g. Análisis de datos se realizó en base a los resultados de las tablas de salida se utilizó como medida la proporción y se establecieron relaciones entre los resultados y la realidad contextual.

h. Redacción de informe final.

\section{MARCO CONCEPTUAL}

En el desarrollo de esta investigación se identificaron algunos conceptos importantes que permiten ir desarrollando la investigación propuesta entre ellos: juventud, PEA, mercado laboral y jornal.

\section{- Juventud}

El período del ciclo de la vida en que las personas transitan de la niñez a la condición adulta, y durante el cual se producen importantes cambios biológicos, psicológicos, sociales y culturales.

Desde el punto de vista demográfico, los jóvenes son ante todo un grupo de población que corresponden a un 
determinado entorno etáreo y varía según los contextos particulares.

Para fines de este estudio se tomaron en cuenta las edades dentro del contexto de juventud la población comprendida entre los 11 a 29 años.

- Población Económicamente Activa (PEA)

La PEA está conformada por las personas que se encuentran ocupadas o buscan trabajo en alguna actividad remunerada y/o orientada a la producción de mercancías (bienes y servicios que serán comercializados en el mercado). La PEI está constituida por aquellas personas que no hacen parte de la PEA (por ejemplo, estudiantes que no trabajan, jubilados, amas de casa, etc.).

- Mercado Laboral

Se denomina mercado de trabajo o mercado laboral al mercado en donde confluyen la demanda y la oferta de trabajo. El mercado de trabajo tiene particularidades que lo diferencian de otro tipo de mercados (financiero, inmobiliario, de comodidades, etc.) ya que se relaciona con la libertad de los trabajadores y la necesidad de garantizar la misma. En ese sentido, el mercado de trabajo suele estar influido y regulado por el Estado a través del derecho laboral y por una modalidad especial de contratos, los convenios colectivos de trabajo.

- Jornal

Forma del salario, que fija la retribución del trabajador a tanto por día de obra.

El jornal agrava los inconvenientes del salario, porque priva al obrero de todo estímulo, exige su vigilancia continua muy costosa y de poco resultado, y hace que, cuando los trabajadores se hallan en común, el esfuerzo se regule por el de los menos diligentes, ya que es igual la retribución de todos.

\section{CONTEXTO GENERAL DEL MUNICIPIO DE CO-} LOMONCAGUA

\subsection{Ubicación Geográfica}

El municipio de Colomoncagua se ubica al sur del departamento de Intibucá, se localiza entre el meridiano $88^{\circ}, 11^{\prime}$ y $88^{\circ} 22^{`}$ longitud oeste y los paralelos $13^{\circ} 7^{`}$ y 14을 longitud norte. Tiene una extensión territorial de $181.42 \mathrm{KM}^{2}$.

Los límites físicos del municipio de Colomoncagua son los siguientes: limita al norte con San Marcos de Sierra y Concepción, al sur con la República de El Salvador, al este con el departamento de La Paz, al oeste con el municipio de Camasca y Magdalena (Ver figura No. 1).

El municipio de Colomoncagua fue fundado el 14 de marzo de 1662 y le dieron su categoría de municipio en 1671, en la actualidad cuenta con 10 aldeas, 134 caseríos (Ver figura No. 2).

El municipio de Colomoncagua se encuentra en un rango de altura que oscila entre 250-900 metros, la temperatura depende de la zona y el comportamiento de los fenómenos naturales.

\subsection{Actividades económicas}

En este municipio las principales actividades económicas son la producción de granos básicos, caña, árboles frutales; además se dedican a la fabricación de teja, jarcia, ladrillo y actividades de tipo comercial sobre todo con la República de El Salvador.

El índice de desempleo aumenta entre los meses de enero hasta abril, época de verano en la cual las activi-

Figura No. 1. Ubicación de Colomononcagua en el Departamento de Intibucá

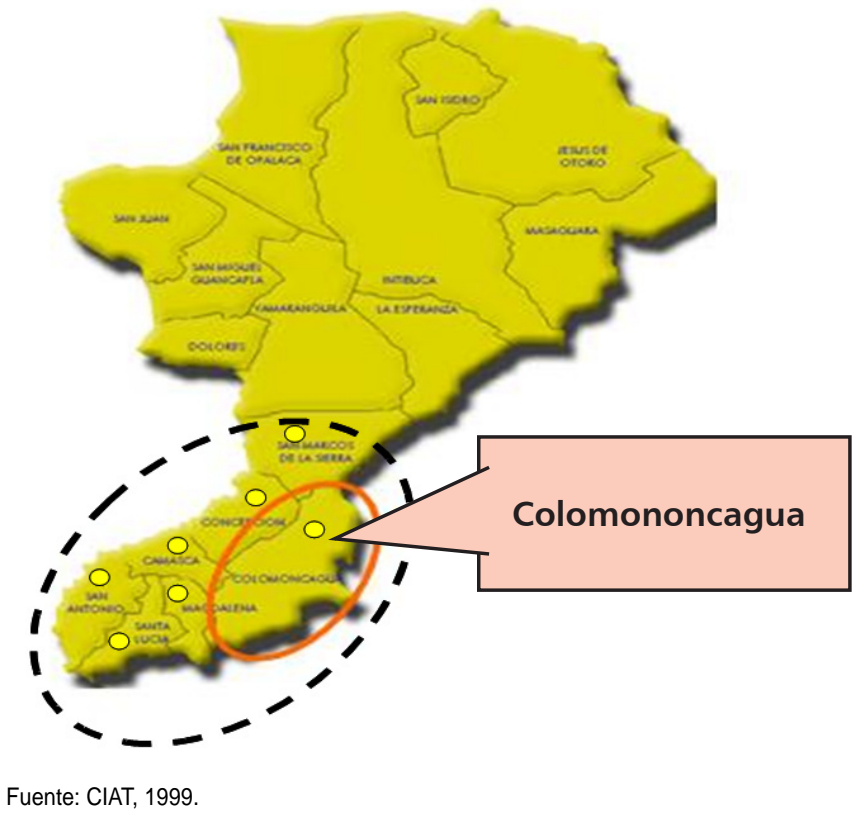


Figura No. 2. Municipio de Colomoncagua por aldeas

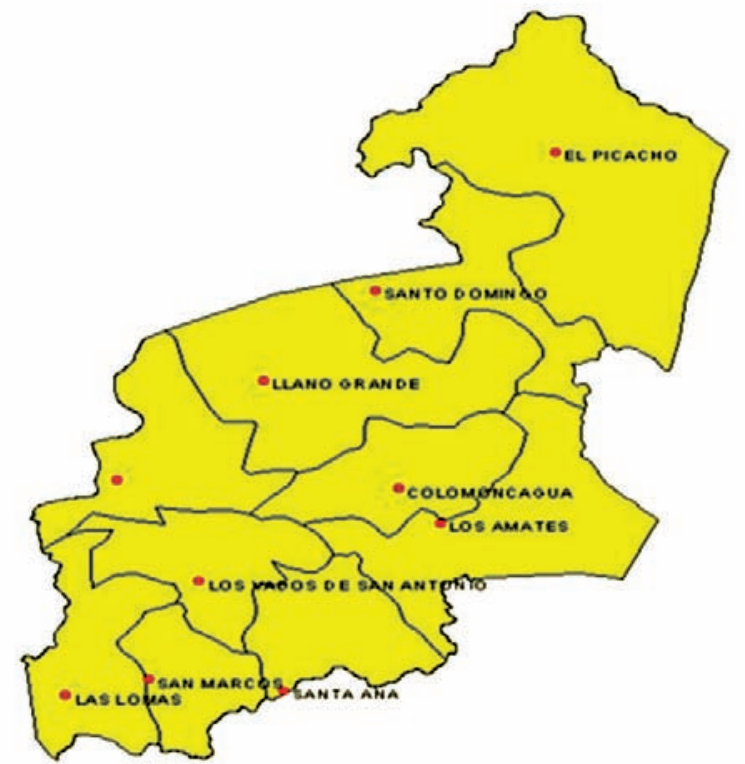

Fuente: SERNA, mapas municipales con división de aldeas

dades agrícolas se detienen temporalmente. De mayo a diciembre se reinicia el cultivo de granos básicos en el que generalmente se emplean los hombres devengando un jornal, las mujeres usualmente se ocupan de las labores propiamente domésticas, pero también están involucradas en actividades comerciales especialmente en la administración de las pulperías y pequeños negocios.

\subsection{Datos Poblacionales}

Según proyecciones de población realizadas por el INE Colomoncagua en el 2009 contaba con una población de 19,223 habitantes de los cuales 50\% son hombres y $50 \%$ mujeres. Colomoncagua es un municipio eminentemente rural.

Se registra un alto crecimiento poblacional de un 3\% anual por lo que esto es una de las causas que influyen a que gran parte de la población del municipio esté conformada por niños, niñas y jóvenes lo que se refleja en la pirámide poblacional del municipio (Ver gráfico No. 1).

\section{SITUACIÓN DE LOS JÓVENES EN EDAD DE TRABAJAR EN EL MUNICIPIO DE COLOMONCAGUA}

El mayor peso poblacional en el municipio de Colomoncagua está representado por la población joven en

\section{Gráfico No. 1. Municipio de Colomoncagua: Pirámide Poblacional}

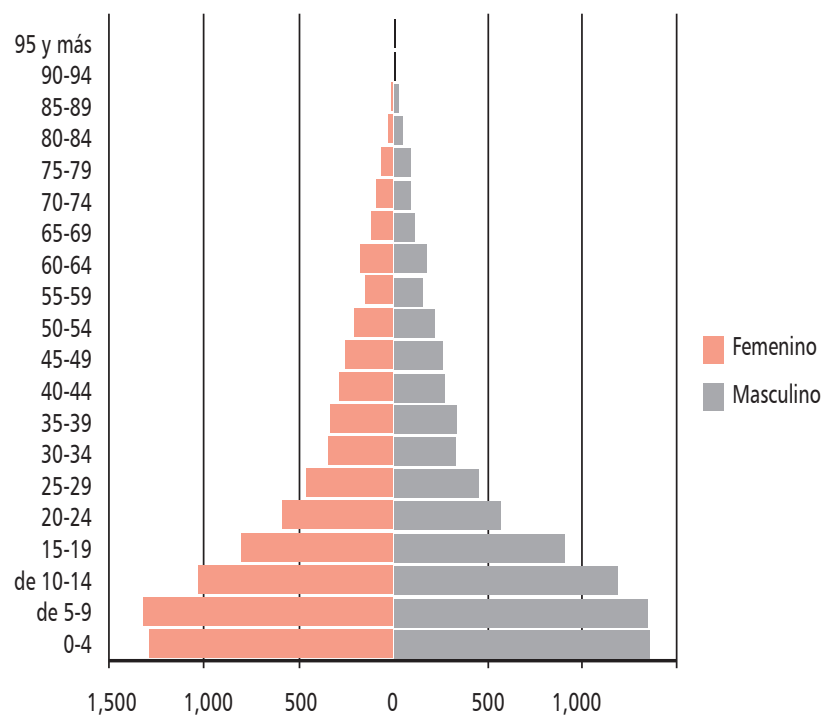

Fuente: Elaboración Propia con datos del Censo de Población y Vivienda 2001.

este apartado se analiza la condición de los jóvenes del municipio en relación a las oportunidades educativas y laborales que se presentan en la zona.

\subsection{Oportunidades Educativas}

La educación constituye uno de los pilares del desarrollo humano permitiendo la formación de capital humano y la ampliación de oportunidades para las personas.

Al caracterizar la población encuestada en relación a su condición de alfabetismo se constató que la mayoría de los jóvenes sabe leer y escribir, el analfabetismo en el municipio se ha reducido y apenas un $5.7 \%$ es analfabeta, y de esta población analfabeta son los hombres los que presentan la mayor proporción. La situación de analfabetismo es más evidente en aldeas como El Picachito, San Antonio y San Miguelito (Dirección Departamental de Educación de Intibucá, 2009).

En el municipio sólo funcionan centros del sistema educativo formal en los niveles de preescolar, educación primaria y educación media, en ese sentido, las oportunidades educativas de los jóvenes se ubican en los niveles de primaria y educación media.

Datos del censo del 2001 registraron que la mayor parte de la población comprendida entre los 11 y 29 
años en el 2001 tenía como nivel escolar la educación primaria, muy pocos tuvieron la oportunidad de continuar con sus estudios de ciclo común y diversificado y apenas el 0.3\% llegó a estudios del nivel superior y universitario (INE, 2001).

Los resultados de la encuesta aplicada en el municipio muestran que la mayor parte de los jóvenes han cursado grados o cursos del nivel primario y del nivel secundario, sin embargo, llama la atención que en ambos niveles un porcentaje considerable tiene incompleto el nivel escolar, para el caso los jóvenes que dejaron su primaria incompleta representan el 21\%, los jóvenes que dejaron incompleto su nivel de ciclo común $26 \%$, y en este grupo son más las mujeres que han dejado incompleto su nivel escolar (Ver cuadro No. 1).

La situación anterior quizá esté relacionada, con la situación de pobreza que enfrentan sus familias, lo que les obliga a insertarse al mercado laboral desde temprana edad, por lo que consecuentemente abandonan los centros escolares.

Entre los jóvenes que lograron terminar su nivel, según sexo no se presenta un comportamiento uniforme, para el caso en el nivel de diversificado son más las mujeres que lograron terminar, en el caso de la primaria completa existe un mayor porcentaje de hombres. En el caso en el que no lograron completar el nivel escolar son las mujeres las que presentan mayor rezago (Ver gráfico No. 2).

\section{Cuadro No. 1. Municipio de Colomoncagua. Nivel Escolar de los Jóvenes}

\begin{tabular}{|l|c|c|}
\hline \multicolumn{1}{|c|}{ Nivel Escolar } & Frecuencia & $\%$ \\
\hline Ninguno & 9 & 4.0 \\
\hline Primaria completa & 30 & 13.2 \\
\hline Primaria incompleta & 49 & 21.6 \\
\hline Ciclo común completo & 34 & 15.0 \\
\hline Ciclo común incompleto & 60 & 26.4 \\
\hline Diversificado completo & 18 & 7.9 \\
\hline Diversificado Incompleto & 15 & 6.6 \\
\hline Universitario completo & 6 & 2.6 \\
\hline Universitario incompleto & 6 & 2.6 \\
\hline Total & 227 & 100.0 \\
\hline
\end{tabular}

Fuente: Elaboración propia, Encuesta Situación de los jóvenes en el Municipio de Colomoncagua, 2009.

\section{Gráfico No. 2. Municipio de Colomoncagua: Nivel Escolar de los Jóvenes, Según Sexo}

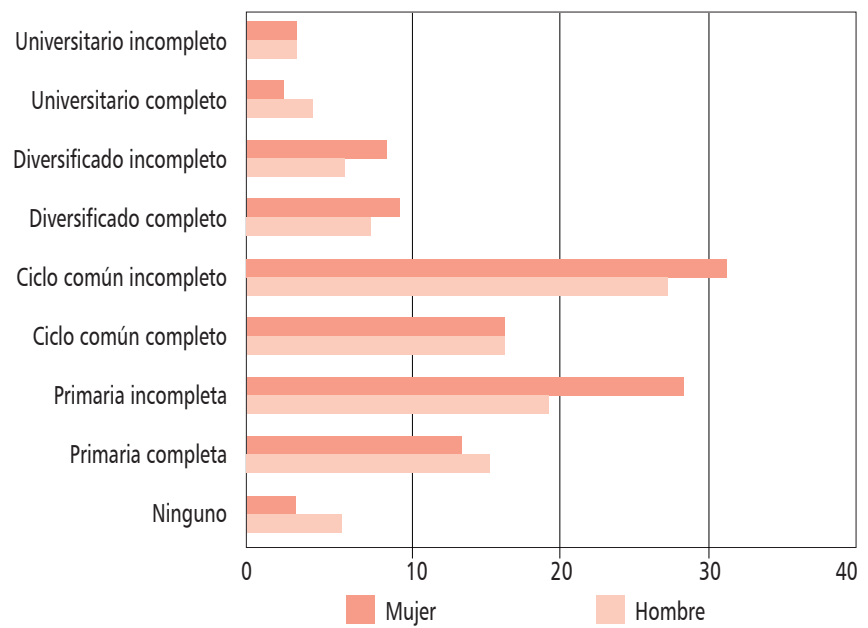

Fuente: Elaboración propia, Encuesta Situación de los jóvenes en el Municipio de Colomoncagua, 2009.

Al desagregar los datos por aldea, se puede observar que los jóvenes que han tenido mayor oportunidad de realizar estudios a nivel universitario son los de la aldea de Colomoncagua y San Miguelito, en tanto que la aldea que concentra el mayor porcentaje de analfabetismo es El Picacho, que se ubica al norte del municipio.

En el nivel de diversificado los y las jóvenes del municipio tienen acceso a carreras como Bachillerato en Administración de Empresas, Bachillerato en Ciencias y Letras y Educación Comercial, por lo que el interés por la preparación académica ha aumentado, sin embargo, no se puede negar gran parte de los y las jóvenes que no tienen acceso a la educación migran principalmente hacia Estados Unidos y hacia la república de El Salvador.

Los y las jóvenes que han estudiado en el nivel universitario generalmente se trasladan hacia las ciudades de Tegucigalpa y San Pedro Sula, lo que ocasiona gastos que no siempre se pueden financiar.

Un detalle que vale la pena resaltar es el hecho de que en la comunidad no existe ningún tipo de academia o taller que les permita a los y las jóvenes de diferentes edades tomar cursos vocacionales de capacitación para desarrollar algunas habilidades y talentos y a la vez generar fuentes de autoempleo. Generalmente los y las jóvenes que desean capacitarse en un oficio, si tienen la oportunidad se trasladan a la ciudad de La Esperanza. 

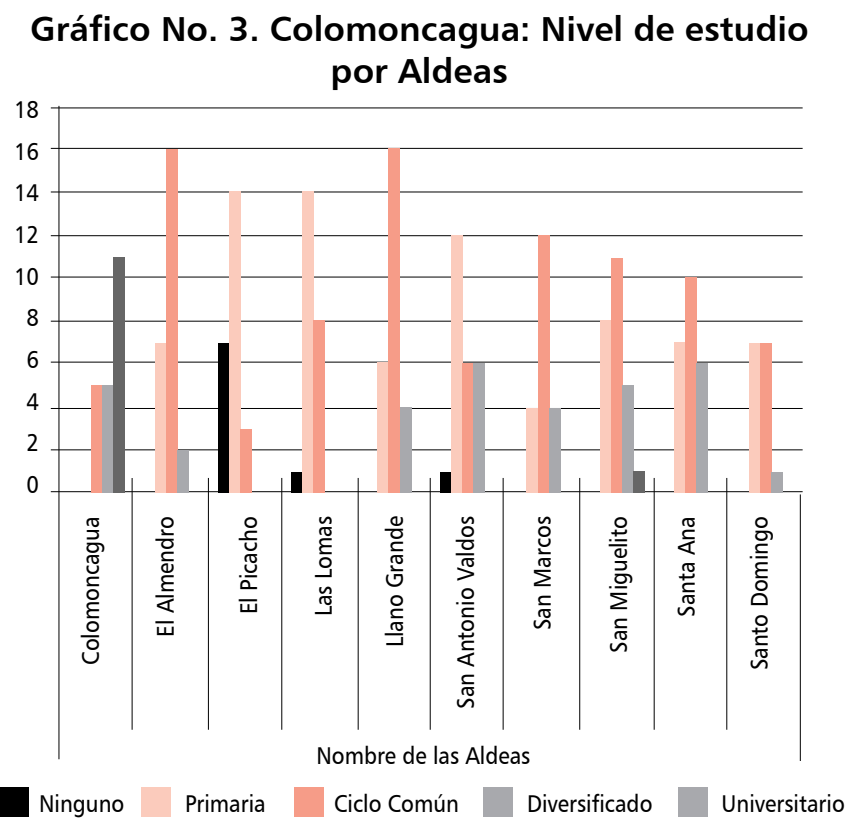

Fuente: Elaboración propia, Encuesta Situación de los jóvenes en el Municipio de Colomoncagua, 2009.

\subsection{Oportunidades Laborales}

Las oportunidades laborales de la población están determinadas principalmente por la estructura del mercado laboral, en ese sentido, a nivel nacional predominan las actividades del sector primario de la economía, y en el caso de Colomoncagua se observa la misma tendencia puesto que la principal actividad económica que se desarrolla en el municipio es la agricultura.

Los trabajos agrícolas al depender directamente de la época de lluvias determinan los períodos de siembra y cosecha, situación que condiciona en gran medida las oportunidades laborales en la comunidad, puesto que la población que está insertado en estas actividades sólo goza de trabajo temporalmente.

Los resultados de la encuesta muestran que El 71\% de los jóvenes entrevistados manifestó estar trabajando, un $20 \%$ trabajaba y estudiaba, $7 \%$ se dedicó exclusivamente a estudiar y el $2 \%$ no realizaba ningún tipo de actividad. Las aldeas que presentan la mayor cantidad de jóvenes trabajando son: El Picachito y Llano Grande, de ahí que en el caso de la primera pueda explicarse en parte el nivel de analfabetismo que existe.

En relación al tipo de actividad que desempeñan según sexo tanto los hombres como las mujeres jóvenes desarrollan como principal actividad laboral la agricultura, ésta no sólo está ligada al trabajo masculino por lo que no se debe desconocer el papel protagónico que juegan las jóvenes. Se cultiva principalmente maíz y frijol que cubren las necesidades de subsistencia de la población; para el uso doméstico se está produciendo

\section{Tabla No. 2. Colomoncagua: Tipo de Actividad según sexo}

\begin{tabular}{|l|c|c|c|}
\hline \multirow{2}{*}{ Tipo de Actividad } & \multicolumn{2}{|c|}{ Sexo del Encuestado } & \multirow{2}{*}{ Total } \\
\cline { 2 - 4 } & Hombre & Mujer & 125 \\
\hline Agricultura & 62 & 63 & 8 \\
\hline Ganadería & 7 & 1 & 19 \\
\hline Comercio & 9 & 10 & 53 \\
\hline Prestación de servicios & 12 & 41 & 22 \\
\hline Construcción & 18 & 4 & 227 \\
\hline Total & 108 & 119 & S \\
\hline
\end{tabular}

Fuente: Elaboración propia, Encuesta Situación de los jóvenes en el Municipio de Colomoncagua, 2009

Tabla No. 3. Municipio de Colomoncagua: Trabajo Infantil

\begin{tabular}{|c|c|c|c|c|c|c|}
\hline & \multicolumn{3}{|c|}{ Tipo de actividad productiva a la que se dedican los jóvenes } & \\
\cline { 2 - 7 } Edad & Agricultura & Ganadería & Comercio & $\begin{array}{c}\text { Prestación de } \\
\text { servicios }\end{array}$ & Construcción & Total \\
\hline 11 & 6 & 0 & 0 & 0 & 1 & 7 \\
\hline 12 & 9 & 0 & 1 & 0 & 0 & 10 \\
\hline 13 & 18 & 0 & 1 & 1 & 2 & 22 \\
\hline 14 & 8 & 0 & 4 & 6 & 1 & 19 \\
\hline 15 & 5 & 1 & 1 & 0 & 2 & 9 \\
\hline 16 & 10 & 2 & 1 & 6 & 1 & 20 \\
\hline 17 & 17 & 0 & 1 & 2 & 1 & 21 \\
\hline Total & 73 & 3 & 9 & 15 & 8 & 108 \\
\hline
\end{tabular}

Fuente: Elaboración propia, Encuesta Situación de los jóvenes en el Municipio de Colomoncagua, 2009 
jabón de aceitunas, estos productos son utilizados para autoconsumo, no hay excedente que permita comercializarlo, quizá esta situación esté ligada al nivel artesanal con el que se desarrollan estas actividades. La segunda actividad de mayor peso es la prestación de servicios en donde están involucradas en mayor proporción las mujeres especialmente en actividades de tipo doméstico y la tercera actividad es la construcción en donde generalmente se involucran más los jóvenes como ayudantes de albañilería. La construcción es una de las actividades que está generando más empleo actualmente, pero carece de mano de obra calificada.

Otro de los elementos que logra rescatarse está relacionado con el trabajo infantil, en el municipio de los 227 encuestados, 108 manifestaron estar trabajando, lo que representa el $48 \%$ de la población, casi la mitad de la población entre 11 y 17 años estaba ocupada en algún tipo de labor que le generaba ingresos a él, ella y su familia.

En relación a los ingresos que estos jóvenes reciben generalmente están muy por debajo del salario mínimo, por ejemplo, la prestación de servicios domésticos es retribuida con un pago de 800 a 1000 lempiras al mes lo que representa un aproximado de 30 lempiras diarios, y el pago de un jornal equivale de 80 a 100 lempiras diarios.

Los y las jóvenes perciben salarios bastante bajos, el $82 \%$ de la población joven encuestada se concentra en el rango de 1000-2000 Lempiras mensuales, esta remuneración generalmente se devenga bajo la modalidad de una forma de pago diario que equivale generalmente a un jornal. Los bajos ingresos que perciben los y las jóvenes apenas solventan sus necesidades básicas limitando cualquier otro tipo de actividades. Es importante mencionar que gran parte de los y las jóvenes laboran con empresas familiares en donde no perciben ningún salario.

Los que reciben los mayores ingresos en el municipio se localizan en la cabecera municipal; estos ingresos provienen de actividades como la prestación de servicios, la construcción y el comercio, en relación a esta última, la posición geográfica de Colomoncagua da lugar al desarrollo de actividades comerciales especialmente con
El Salvador, sin embargo, son muy pocos los jóvenes que están involucrados en estas actividades puesto que requieren de capital que en la mayoría de las ocasiones es inaccesible para esta población.

Otro detalle que vale la pena resaltar es que esta población trabaja sin ningún beneficio adicional como pago de vacaciones, días feriados, aguinaldos etc, esta situación es similar a la del resto del país sobre todo en el área rural.

\section{Tabla No. 4. Municipio de Colomoncagua: Monto Salarial que devenga la población joven}

\begin{tabular}{|l|c|c|}
\hline \multicolumn{1}{|c|}{ Monto Salarial } & Frecuencia & $\%$ \\
\hline$<1000$ & 20 & 8.8 \\
\hline $1000-2000$ & 188 & 82.8 \\
\hline $2001-3000$ & 15 & 6.6 \\
\hline $3001-4000$ & 4 & 1.8 \\
\hline Total & 227 & 100.0 \\
\hline
\end{tabular}

Fuente: Elaboración propia, Encuesta Situación de los jóvenes en el Municipio de Colomoncagua, 2009.

\section{CONCLUSIONES}

- Las oportunidades educativas de la población joven en el municipio son bastante limitadas, pues aunque el sistema educativo formal da cobertura a los niveles de educación primaria y secundaria gran parte de la población se encuentra en situación de pobreza limitando las oportunidades de acceso a la educación a niños, niñas y jóvenes del municipio que desde muy temprana edad se insertan en actividades laborales que les permite el acceso a ingresos económicos.

- La mayoría de los jóvenes que trabajan en el municipio de Colomoncagua devenga salarios entre 1000 a 2000 Lempiras muy por debajo del salario mínimo establecido en el área rural, sin embargo, las escasas oportunidades de empleo los hacen permanecer en ellos, además una buena parte de niños, niñas y jóvenes realizan trabajos con sus familias los cuales no son remunerados.

- No puede desconocerse que en el municipio de Colomoncagua existe un buen porcentaje de trabajo infantil, esta situación incide desfavorablemente en el desarrollo integral del niño o niña y en su formación aca- 
démica puesto que la mayoría de ellos tenía su primaria incompleta o su ciclo común incompleto.

- Los y las jóvenes que trabajan en el municipio de Colomoncagua lo hacen en actividades como la agricultura, la prestación de servicios y la construcción, generalmente percibiendo salarios por debajo de mínimo establecido en el área rural, esto limita sus oportunidades de acceso a la educación, salud, recreación y a la vez su oportunidad de desarrollo humano.

- El municipio de Colomoncagua no cuenta con ningún tipo de institución del sector formal e informal que pueda brindar las oportunidades de capacitación de los jóvenes en oficios que les permitan establecer a futuro pequeñas microempresas.

IX-BIBLIOGRAFÍA

- INE, 2001, base de datos del censo del 2001.

- $\quad$ SERNA mapas municipales con división de aldeas, 2006.

- Plan Estratégico de la Municipalidad de Colomoncagua, 2007.

- Encuesta Situación de los jóvenes en el Municipio de Colomoncagua, 2009. 